\title{
843.
}

\section{ON THE QUADRIQUADRIC CURVE IN CONNEXION WITH THE THEORY OF ELLIPTIC FUNCTIONS.}

[From the Mathematische Annalen, t. xxv. (1885), pp. 152-156.]

I CALL to mind that, if we have on a line two points $(\alpha, \beta, \gamma, \delta),\left(\alpha^{\prime}, \beta^{\prime}, \gamma^{\prime}, \delta^{\prime}\right)$, and through the line two planes

then

$$
A x+B y+C z+D w=0, \quad A^{\prime} x+B^{\prime} y+C^{\prime} z+D^{\prime} w=0,
$$

$$
\begin{aligned}
& \beta \gamma^{\prime}-\beta^{\prime} \gamma: \gamma \alpha^{\prime}-\gamma^{\prime} \alpha: \alpha \beta^{\prime}-\alpha^{\prime} \beta: \alpha \delta^{\prime}-\alpha^{\prime} \delta: \beta \delta^{\prime}-\beta^{\prime} \delta: \gamma \delta^{\prime}-\gamma^{\prime} \delta \\
= & A D^{\prime}-A^{\prime} D: B D^{\prime}-B^{\prime} D: C D^{\prime}-C^{\prime} D: B C^{\prime}-B^{\prime} C: C A^{\prime}-C^{\prime} A: A B^{\prime}-A^{\prime} B ;
\end{aligned}
$$

and that, putting each of these two equal sets of ratios

$$
=a: b: c: f: g: h \text {, }
$$

then the quantities $(a, b, c, f, g, h)$, which it is easy to see satisfy the relation $a f+b g+c h=0$, are said to be the "six coordinates" of the line: as only the ratios of the six quantities are material, and as the last-mentioned equation establishes a single relation between these ratios, the system of the six coordinates contains four arbitrary ratios or parameters, for the determination of the particular line. See my paper "On the six coordinates of a line," Camb. Phil. Trans. t. xi. (1869), pp. 290-323, [435].

I consider for a moment the quadric surface

$$
x^{2}+y^{2}+z^{2}+w^{2}=0,
$$

and I proceed to show that, if $(a, b, c, f, g, h)$ are the coordinates of a generating line on the surface, then either $a=f, b=g, c=h$, or else $a=-f, b=-g, c=-h$; the one or other system of equations according as the line belongs to the one or other system of generating lines.

c. XII. 
We satisfy the equation by

$$
\begin{aligned}
& x+i y+\theta z+\theta i w=0 \\
& x-i y-\frac{1}{\theta} z+\frac{1}{\theta} i w=0
\end{aligned}
$$

where $\theta$ is an arbitrary parameter: hence these equations determine a generating line of the surface: and the coordinates of this line are

$$
\begin{aligned}
& a, \quad b, \quad-\quad c, \quad f, \quad g, \quad h \\
& =-i\left(\theta-\frac{1}{\theta}\right),-\left(\theta+\frac{1}{\theta}\right), z i, i\left(\theta-\frac{1}{\theta}\right), \theta+\frac{1}{\theta},-z i \text {; }
\end{aligned}
$$

viz. these values give $a=-f, b=-g, c=-h$.

Similarly we satisfy the equation by

$$
\begin{aligned}
& x+i y+\phi z-\phi i w=0, \\
& x-i y-\frac{1}{\phi} z-\frac{1}{\phi} i w=0
\end{aligned}
$$

where $\phi$ is an arbitrary parameter: hence these equations also determine a generating line of the surface: and the coordinates of this line are

$$
\begin{array}{cccccc}
a, & b, & c, & f, & g, & h \\
=i\left(\phi-\frac{1}{\phi}\right), & \phi+\frac{1}{\phi}, & -z i, & i\left(\phi+\frac{1}{\phi}\right), & \phi+\frac{1}{\phi}, & -z i ;
\end{array}
$$

viz. these values give $a=f, b=g, c=h$.

If for $x, y, z, w$ we write $x \sqrt{p}, y \sqrt{q}, z \sqrt{r}, w \sqrt{s}$ respectively, then we have the theorem that, for the quadric surface

$$
p x^{2}+q y^{2}+r z^{2}+s w^{2}=0,
$$

the coordinates $(a, b, c, f, g, h)$ of a generating line are such that

$$
\frac{a}{f}= \pm \sqrt{\frac{p s}{q r}}, \quad \frac{b}{g}= \pm \sqrt{\frac{q s}{r p}}, \quad \frac{c}{h}= \pm \sqrt{\frac{r s}{p q}},
$$

the signs being all + or all -, according as the line belongs to one or other of the systems of generating lines.

Take $\left(a^{\prime}, b^{\prime}, c^{\prime}, f^{\prime}, g^{\prime}, h^{\prime}\right)$ for the coordinates of an arbitrary line, and write $p, q, r, s=a^{\prime} g^{\prime} h^{\prime}, b^{\prime} h^{\prime} f^{\prime}, c^{\prime} f^{\prime} g^{\prime}, a^{\prime} b^{\prime} c^{\prime}$; the quadric surface is

$$
a^{\prime} g^{\prime} h^{\prime} x^{2}+b^{\prime} h^{\prime} f^{\prime} y^{2}+c^{\prime} f^{\prime} g^{\prime} z^{2}+a^{\prime} b^{\prime} c^{\prime} w^{2}=0
$$


which is a surface having the line $\left(a^{\prime}, b^{\prime}, c^{\prime}, f^{\prime}, g^{\prime}, h^{\prime}\right)$ for a generating line. To verify this, observe that for the line in question we have

$$
\begin{array}{r}
h^{\prime} y-g^{\prime} z+a^{\prime} w=0 \\
-h^{\prime} x+f^{\prime} z+b^{\prime} w=0 \\
g^{\prime} x+f^{\prime} y+c^{\prime} w=0 \\
-a^{\prime} x-b^{\prime} y-c^{\prime} z \quad=0
\end{array}
$$

equivalent of course to two independent equations: these give $h^{\prime} x=f^{\prime} z+b^{\prime} w$, $h^{\prime} y=g^{\prime} z-a^{\prime} w$, values which substituted in the quadric equation satisfy it identically. And for the last-mentioned quadric surface we have the theorem that, if $(a, b, c, f, g, h)$ are the coordinates of a generating line, then

$$
\frac{a}{f}= \pm \frac{a^{\prime}}{f^{\prime}}, \quad \frac{b}{g}= \pm \frac{b^{\prime}}{g^{\prime}}, \quad \frac{c}{h}= \pm \frac{c^{\prime}}{h^{\prime}},
$$

where obviously the sign + belongs to a generating line of the same system with the line $\left(a^{\prime}, b^{\prime}, c^{\prime}, f^{\prime}, g^{\prime}, h^{\prime}\right)$, and the sign - to a line of the other system.

Taking the sign -, we thus see that if $(a, b, c, f, g, h),\left(a^{\prime}, b^{\prime}, c^{\prime}, f^{\prime}, g^{\prime}, h^{\prime}\right)$ are the coordinates of lines of the two systems respectively, then

$$
a f^{\prime}+a^{\prime} f=0, \quad b g^{\prime}+b^{\prime} g=0, \quad c h^{\prime}+c^{\prime} h=0 ;
$$

where observe that the resulting equation

$$
a f^{\prime}+a^{\prime} f+b g^{\prime}+b^{\prime} g+c h^{\prime}+c^{\prime} h=0,
$$

is the condition which expresses that the two lines meet each other.

Consider now the quadriquadric curve

$$
\begin{aligned}
& U_{1}=A x^{2}+B y^{2}+C z^{2}+D w^{2}=0 \\
& U_{1}^{\prime}=A^{\prime} x^{2}+B^{\prime} y^{2}+C^{\prime} z^{2}+D^{\prime} w^{2}=0
\end{aligned}
$$

and let $(a, b, c, f, g, h)$ and $\left(a^{\prime}, b^{\prime}, c^{\prime}, f^{\prime}, g^{\prime}, h^{\prime}\right)$ be the coordinates of two lines meeting each other, and each meeting the quadric curve twice: or, again, let these be lines joining in pairs the four intersections of the curve by an arbitrary plane: or, again, let them be the nodal lines of the binodal quartic cone having an arbitrary vertex and passing through the curve. The two lines are generating lines, belonging to the two systems respectively, of a properly determined quadric surface $U+\lambda U^{\prime}=0$ passing through the curve: and by what precedes, we have

$$
a f^{\prime}+a^{\prime} f=0, \quad b g^{\prime}+b^{\prime} g=0, \quad c h^{\prime}+c^{\prime} h=0,
$$

the fundamental theorem "which I wished to establish.

Writing in the equations $w=1$, we have in particular the quadriquadric curvo $y^{2}=1-x^{2}, z^{2}=1-k^{2} x^{2}$, equations which are satisfied by $x=\operatorname{sn} u, y=\operatorname{cn} u, z=\operatorname{dn} u$. Consider on the curve four points belonging to the arguments $u_{1}, u_{2}, u_{3}, u_{4}$ respectively; 
and write for shortness $s_{1}, c_{1}, d_{1}$ for the sn, en, and $d n$ of $u_{1}$; and similarly for $u_{2}, u_{3}$ and $u_{4}$. It appears by Abel's theorem that the condition in order that the four points may be in a plane is $u_{1}+u_{2}+u_{3}+u_{4}=0$; viz. when this equation is satisfied we have

or writing

$$
\left|\begin{array}{llll}
s_{1}, & c_{1}, & d_{1}, & 1 \\
s_{2}, & c_{2}, & d_{2}, & 1 \\
s_{3}, & c_{3}, & d_{3}, & 1 \\
s_{4}, & c_{4}, & d_{4}, & 1
\end{array}\right|,
$$

$$
\begin{array}{cccccc}
a, & b, & c, & f, & g, & h \\
=c_{1} d_{2}-c_{2} d_{1}, & d_{1} s_{2}-d_{2} s_{1}, & s_{1} c_{2}-s_{2} c_{1}, & s_{1}-s_{2}, & c_{1}-c_{2}, & d_{1}-d_{2}, \\
a^{\prime}, & b^{\prime}, & c^{\prime}, & f^{\prime}, & g^{\prime}, & h^{\prime} \\
=c_{3} d_{4}-c_{4} d_{3}, & d_{3} s_{4}-d_{4} s_{3}, & s_{3} c_{4}-s_{4} c_{3}, & s_{3}-s_{4}, & c_{3}-c_{4}, & d_{3}-d_{4},
\end{array}
$$

this equation is

$$
a f^{\prime}+a^{\prime} f+b g^{\prime}+b^{\prime} g+c h^{\prime}+c^{\prime} h=0
$$

or by what precedes, it appears that not only is this so, but that we have separately

$$
a f^{\prime}+a^{\prime} f=0, \quad b g^{\prime}+b^{\prime} g=0, \quad c h^{\prime}+c^{\prime} h=0,
$$

viz. it follows from Abel's theorem that, when the arguments $u_{1}, u_{2}, u_{3}, u_{4}$ are connected by the equation $u_{1}+u_{2}+u_{3}+u_{4}=0$, then each of these three equations holds good.

I assume in particular $u_{4}=0, u_{3}=-u$, so that $u=u_{1}+u_{2}$; we have

$$
\begin{aligned}
& \begin{array}{lllll}
a, & b, & c &
\end{array}, \quad g, \quad h \\
& =c_{1} d_{2}-c_{2} d_{1}, \quad d_{1} s_{2}-d_{2} s_{1}, \quad s_{1} c_{2}-s_{2} c_{1}, \quad s_{1}-s_{2}, \quad c_{1}-c_{2}, \quad d_{1}-d_{2} \text {, } \\
& a^{\prime}, \quad b^{\prime}, \quad c^{\prime}, \quad f^{\prime}, \quad g^{\prime}, \quad h^{\prime} \\
& =c-d, \quad s, \quad-s, \quad-s, \quad c-1, \quad d-1,
\end{aligned}
$$

and the three equations become

$$
\frac{c-d}{s}=\frac{c_{1} d_{2}-c_{2} d_{1}}{s_{1}-s_{2}}, \quad \frac{-s}{c-1}=\frac{d_{1} s_{2}-d_{2} s_{1}}{c_{1}-c_{2}}, \quad \frac{s}{d-1}=\frac{s_{1} c_{2}-s_{2} c_{1}}{d_{1}-d_{2}},
$$

equations which may also be written

$$
\frac{c-d}{s}=\frac{c_{1} d_{2}-c_{2} d_{1}}{s_{1}-s_{2}}, \quad \frac{-c+1}{s}=\frac{c_{1}-c_{2}}{d_{1} s_{2}-d_{2} s_{1}}, \quad \frac{d-1}{s}=\frac{d_{1}-d_{2}}{s_{1} c_{2}-s_{2} c_{1}},
$$

so that, adding these three equations, we must have an identity.

Representing the second and third of the last-mentioned equations by

$$
\frac{-c+1}{s}=C, \quad \frac{d-1}{s}=D,
$$


we have

$$
c=1-C s, \quad d=1+D s,
$$

from either of which we can obtain $s$ rationally; viz. the first equation gives

$$
1-s^{2}=1-2 C s+C^{2} s^{2}
$$

that is, $s=\frac{2 C}{1+C^{2}}$, whence

$$
c=\frac{1-C^{2}}{1+C^{2}}, \quad d=\frac{1+C^{2}+2 C D}{1+C^{2}}
$$

and similarly from the second equation $s=-\frac{2 D}{k^{2}+D^{2}}$, whence also

$$
c=\frac{k^{2}+D^{2}-2 C D}{k^{2}+D^{2}}, \quad d=\frac{k^{2}-D^{2}}{k^{2}+D^{2}} .
$$

Substituting for $C$ its value, the first-mentioned value of $s$ becomes

$$
s=\frac{\left(c_{1}-c_{2}\right)\left(d_{1} s_{2}-d_{2} s_{1}\right)}{1-k^{2} s_{1}^{2} s_{2}^{2}-c_{1} c_{2}-d_{1} s_{1} d_{2} s_{2}},
$$

which is a form that can be easily verified: we in fact have

$$
s_{1}=\operatorname{sn}\left(u_{1}+u_{2}\right)=\frac{s_{1} c_{2} d_{2}+s_{2} c_{1} d_{1}}{1-k^{2} s_{1}{ }^{2} s_{2}{ }^{2}},=\frac{s_{1}{ }^{2}-s_{2}{ }^{2}}{s_{1} c_{2} d_{2}-s_{2} c_{1} d_{1}},=\frac{s_{1} c_{1} d_{2}+s_{2} c_{2} d_{1}}{c_{1} c_{2}+s_{1} d_{1} s_{2} d_{2}},=\frac{s_{1} d_{1} c_{2}+s_{2} d_{2} c_{1}}{d_{1} d_{2}+k^{2} s_{1} c_{1} s_{2} c_{2}},
$$

see my Elliptic Functions, p. 63: or calling these values

$$
=\frac{N_{1}}{D_{1}}=\frac{N_{2}}{D_{2}}=\frac{N_{3}}{D_{3}}=\frac{N_{4}}{D_{4}},
$$

the above value is

$$
s=\frac{N_{1}-N_{3}}{D_{1}-D_{3}}
$$

which is right.

Cambridge, 28 June, 1884. 